\title{
Repetition Avoidance in Circular Factors
}

\author{
Hamoon Mousavi and Jeffrey Shallit \\ School of Computer Science, University of Waterloo, Waterloo, ON N2L 3G1 Canada \\ \{sh2mousa, shallit\}@uwaterloo.ca
}

\begin{abstract}
We consider the following novel variation on a classical avoidance problem from combinatorics on words: instead of avoiding repetitions in all factors of a word, we avoid repetitions in all factors where each individual factor is considered as a "circular word", i.e., the end of the word wraps around to the beginning. We determine the best possible avoidance exponent for alphabet size 2 and 3, and provide a lower bound for larger alphabets.
\end{abstract}

\section{Introduction}

Repetition in words is an active research topic and has been studied for over a hundred years. For example, Axel Thue [1011] constructed an infinite word over a three-letter alphabet that contains no squares (i.e., no nonempty word of the form $x x$ ), and another infinite word over a two-letter alphabet that contains no cubes (i.e., no nonempty word of the form $x x x$ ).

In 1972, Dejean refined these results by considering fractional powers. An $\alpha$-power for a rational number $\alpha \geq 1$ is a word of the form $w=x^{\lfloor\alpha\rfloor} x^{\prime}$, where $x^{\prime}$ is a (possibly empty) prefix of $x$ and $|w|=\alpha|x|$. The word $w$ is a repetition, with a period $x$ and an exponent $\alpha$. Among all possible exponents, we let $\exp (w)$ denote the largest one, corresponding to the shortest period. For example, the word alfalfa has shortest period alf and exponent $\frac{7}{3}$. The critical exponent of a word $w$ is the supremum, over all factors $f$ of $w$, of $\exp (f)$. We write it as $\exp (w)$.

For a real number $\alpha$, an $\alpha^{+}$-power is a $\beta$-power where $\beta>\alpha$. For example $a b a b a=(a b)^{\frac{5}{2}}$ is a $2^{+}$-power. A word $w$ is

$-\alpha^{+}$-power-free, if none of the factors of $w$ is an $\alpha^{+}$-power;

$-\alpha$-power-free if, in addition to being $\alpha^{+}$-power-free, the word $w$ has no factor that is an $\alpha$-power.

We also say that $w$ avoids $\alpha^{+}$-powers (resp., avoids $\alpha$-powers). Dejean asked, what is the smallest real number $r$ for which there exist infinite $r^{+}$-power-free words over an alphabet of size $k$ ? This quantity is called the repetition threshold [2], and is denoted by $\mathrm{RT}(k)$. From results of Thue we know that $\mathrm{RT}(2)=2$. Dejean [5] proved RT(3) $=\frac{7}{4}$, and conjectured that

$$
\operatorname{RT}(k)= \begin{cases}\frac{7}{5}, & \text { if } k=4 \\ \frac{k}{k-1}, & \text { if } k>4\end{cases}
$$


This conjecture received much attention in the last forty years, and its proof was recently completed by Currie and Rampersad [4] and Rao [9, independently, based on work of Carpi [3] and others.

We consider the following novel variation on Dejean, which we call "circular $\alpha$-power avoidance". We consider each finite factor $x$ of a word $w$, but interpret such a factor as a "circular" word, where the end of the word wraps around to the beginning. Then we consider each factor $f$ of this interpretation of $x$; for $w$ to be circularly $\alpha$-power-free, each such $f$ must be $\alpha$-power-free. For example, consider the English word $w=$ dividing with factor $x=$ dividi. The circular shifts of $x$ are

$$
\text { dividi, ividid, vididi, ididiv, didivi, idivid, }
$$

and (for example) the word ididiv contains a factor ididi that is a $\frac{5}{2}$-power. In fact, $w$ is circularly cubefree and circularly $\left(\frac{5}{2}\right)^{+}$-power-free.

To make this more precise, we recall the notion of conjugacy. Two words $x, y$ are conjugate if one is a cyclic shift of the other; that is, if there exist words $u, v$ such that $x=u v$ and $y=v u$.

Definition 1. Let $w$ be a finite or infinite word. The largest circular $\alpha$-power in a word $w$ is defined to be the supremum of $\exp (f)$ over all factors $f$ of conjugates of factors of $w$. We write it as $\operatorname{cexp}(w)$.

Although Definition 1 characterizes the subject of this paper, we could have used a different definition, based on the following.

Proposition 2. Let $w$ be a finite word or infinite word. The following are equivalent:

(a) $s$ is a factor of a conjugate of a factor of $w$;

(b) $s$ is a prefix of a conjugate of a factor of $w$;

(c) $s$ is a suffix of a conjugate of a factor of $w$;

(d) $s=v t$ for some factor tuv of $w$.

Proof. (a) $\Longrightarrow$ (b): Suppose $s=y^{\prime \prime} x^{\prime}$, where $x y$ is a factor of $w$ and $x=x^{\prime} x^{\prime \prime}$ and $y=y^{\prime} y^{\prime \prime}$. Another conjugate of $x y$ is then $y^{\prime \prime} x^{\prime} x^{\prime \prime} y^{\prime}$ with prefix $y^{\prime \prime} x^{\prime}$.

(b) $\Longrightarrow(\mathrm{c})$ : Such a prefix $s$ is either of the form $y^{\prime}$ or $y x^{\prime}$, where $x y$ be a factor of $w$ and $x=x^{\prime} x^{\prime \prime}$ and $y=y^{\prime} y^{\prime \prime}$. Considering the conjugate $y^{\prime \prime} x y^{\prime}$ of $y x$, we get a suffix $y^{\prime}$, and consider the conjugate $x^{\prime \prime} y x^{\prime}$ we get a suffix $y x^{\prime}$.

$(c) \Longrightarrow(d)$ : Such a suffix $s$ is either of the form $s=x^{\prime \prime}$ or $s=y^{\prime \prime} x$, where $x y$ be a factor of $w$ and $x=x^{\prime} x^{\prime \prime}$ and $y=y^{\prime} y^{\prime \prime}$. In the former case, let $t=x^{\prime \prime}$, $u=v=\epsilon$. In the latter case, let $t=x, u=y^{\prime}$, and $v=y^{\prime \prime}$.

$(\mathrm{d}) \Longrightarrow(\mathrm{a})$ : Let $t u v$ be a factor of $w$. Then $v t u$ is a conjugate of $t u v$, and $v t$ is a factor of it.

Let $\Sigma_{k}=\{0,1, \ldots, k-1\}$. Define RTC $(k)$, the repetition threshold for circular factors, to be the smallest real number $r$ for which there exist infinite circularly $r^{+}$-power-free words in $\Sigma_{k}$. Clearly we have

$$
\mathrm{RTC}(k) \geq \mathrm{RT}(k) \text {. }
$$


In this paper we prove that $\operatorname{RTC}(2)=4$ and $\operatorname{RTC}(3)=\frac{13}{4}$. For larger alphabets, we conjecture that

$$
\operatorname{RTC}(k)= \begin{cases}\frac{5}{2}, & \text { if } k=4 \\ \frac{105}{46}, & \text { if } k=5 \\ \frac{2 k-1}{k-1}, & \text { if } k \geq 6 .\end{cases}
$$

Finally, we point out that the quantities we study here are not closely related to the notion of avoidance in circular words, studied previously in [16]7.

Acknowledgments. We thank the referees for their careful reading of this paper.

\section{Notation}

For a finite alphabet $\Sigma$, let $\Sigma^{*}$ denote the set of finite words over $\Sigma$. Let $\Sigma^{\omega}$ denote the set of right infinite words over $\Sigma$, and let $\Sigma^{\infty}=\Sigma^{\omega} \cup \Sigma^{*}$. Let $w=a_{0} a_{1} \cdots \in \Sigma^{\infty}$ be a word. Let $w[i]=a_{i}$, and let $w[i . . j]=a_{i} \cdots a_{j}$. By convention we have $w[i]=\epsilon$ for $i<0$ and $w[i . . j]=\epsilon$ for $i>j$. Note that if $x$ is a period of $w$ and $|x|=p$, then $w[i+p]=w[i]$ for $0 \leq i<|w|-p$.

For a word $x$, let $\operatorname{pref}(x)$ and suff $(x)$, respectively, denote the set of prefixes and suffixes of $x$. For words $x, y$, let $x \preceq y$ denote that $x$ is a factor of $y$. Let $x \preceq_{p} y$ (resp., $x \preceq_{s} y$ ) denote that $x$ is a prefix (resp., suffix) of $y$.

A morphism $h: \Sigma^{*} \rightarrow \Phi^{*}$ is said to be $q$-uniform if $|h(a)|=q$ for all $a \in \Sigma$. A morphism is uniform if it is $q$-uniform for some $q$. The fixed point of a morphism $h: \Sigma^{*} \rightarrow \Phi^{*}$ starting with $a \in \Sigma$, if it exists, is denoted by $h^{\omega}(a)$.

In the next section, we prove some preliminary results. We get some bounds for $\operatorname{RTC}(k)$, and in particular, we prove that $\mathrm{RTC}(2)=2 \mathrm{RT}(2)=4$. In Section 4. we study the three-letter alphabet, and we prove that $\operatorname{RTC}(3)=\frac{13}{4}$. Finally, in Section [5 we give another interpretation for repetition threshold for circular factors.

\section{Binary Alphabet}

First of all, we prove a bound on $\operatorname{RTC}(k)$.

Theorem 3. $1+\mathrm{RT}(k) \leq \mathrm{RTC}(k) \leq 2 \mathrm{RT}(k)$.

Proof. Let $r=\mathrm{RT}(k)$. We first prove that $\mathrm{RTC}(k) \leq 2 r$. Let $w \in \Sigma_{k}^{\omega}$ be an $r^{+}$power-free word. We prove that $w$ is circularly $(2 r)^{+}$-power-free. Suppose that $x t y \preceq w$, such that $y x$ is $(2 r)^{+}$-power. Now either $y$ or $x$ is an $r^{+}$-power. This implies that $w$ contains an $r^{+}$-power, a contradiction.

Now we prove that $1+r \leq \operatorname{RTC}(k)$. Let $l$ be the length of the longest $r$ power-free word over $\Sigma_{k}$, and let $w \in \Sigma_{k}^{\omega}$. Considering the factors of length $n=l+1$ of $w$, we know some factor $f$ must occur infinitely often. This $f$ contains an $r$-power: $z^{r}$. Therefore $z^{r} t z$ is a factor of $w$. Therefore $w$ contains a circular $(1+r)$-power. This proves that $1+r \leq \mathrm{RTC}(k)$. 
Note that since $\mathrm{RT}(k)>1$, we have $\mathrm{RTC}(k)>2$.

Lemma 4. $\mathrm{RTC}(2) \geq 4$.

Proof. Let $w \in \Sigma_{2}^{\omega}$ be an arbitrary word. It suffices to prove that $w$ contains circular 4-powers. There are two cases: either 00 or 11 appears infinitely often, or $w$ ends with a suffix of the form $(01)^{\omega}$. In the latter case, obviously there are circular 4-powers; in the former there are words of the form aayaa for $a \in \Sigma_{2}$ and $y \in \Sigma_{2}^{*}$ and hence circular 4 -powers.

Theorem 5. $\mathrm{RTC}(2)=4$.

Proof. A direct consequence of Theorem 3 and Lemma 4 .

The Thue-Morse word is an example of a binary word that avoids circular $4^{+}$-powers.

\section{Ternary Alphabet}

Our goal in this section is to show that $\operatorname{RTC}(3)=\frac{13}{4}$. For this purpose, we frequently use the notion of synchronizing morphism, which was introduced in Ilie et al. [8].

Definition 6. A morphism $h: \Sigma^{*} \rightarrow \Gamma^{*}$ is said to be synchronizing if for all $a, b, c \in \Sigma$ and $s, r \in \Gamma^{*}$, if $h(a b)=r h(c) s$, then either $r=\epsilon$ and $a=c$ or $s=\epsilon$ and $b=c$.

Definition 7. A synchronizing morphism $h: \Sigma^{*} \rightarrow \Gamma^{*}$ is said to be strongly synchronizing if for all $a, b, c \in \Sigma$, if $h(c) \in \operatorname{pref}(h(a)) \operatorname{suff}(h(b))$, then either $c=a$ or $c=b$.

The following technical lemma is applied several times throughout the paper.

Lemma 8. Let $h: \Sigma^{*} \rightarrow \Gamma^{*}$ be a synchronizing q-uniform morphism. Let $n>1$ be an integer, and let $w \in \Sigma^{*}$. If $z^{n} \preceq_{p} h(w)$ and $|z| \geq q$, then $u^{n} \preceq_{p} w$ for some $u$. Furthermore $|z| \equiv 0(\bmod q)$.

Proof. Let $z=h(u) z^{\prime}$, where $\left|z^{\prime}\right|<q$ and $u \in \Sigma^{*}$. Note that $u \neq \epsilon$, since $|z| \geq q$. Clearly, we have $z^{\prime} h(u[0]) \preceq_{p} h(w[|u| . .|u|+1])$. Since $h$ is synchronizing, the only possibility is that $z^{\prime}=\epsilon$, so $|z| \equiv 0(\bmod q)$. Now we can write $z^{n}=h\left(u^{n}\right) \preceq_{p}$ $h(w)$. Therefore $u^{n} \preceq_{p} w$.

The next lemma states that if the fixed point of a strongly synchronizing morphism (SSM) avoids small $n$ 'th powers, where $n$ is an integer, it avoids $n$ 'th powers of all lengths.

Lemma 9. Let $h: \Sigma^{*} \rightarrow \Sigma^{*}$ be a strongly synchronizing q-uniform morphism. Let $n>1$ be an integer. If $h^{\omega}(0)$ avoids factors of the form $z^{n}$, where $\left|z^{n}\right|<2 n q$, then $h^{\omega}(0)$ avoids $n$ 'th powers. 
Proof. Let $w=a_{0} a_{1} a_{2} \cdots=h^{\omega}(0)$. Suppose $w$ has $n$ 'th powers of length greater than or equal to $2 n q$. Let $z$ be the shortest such word, i.e., $\left|z^{n}\right| \geq 2 n q$ and $z^{n} \preceq w$. We can write

$$
\begin{aligned}
& z^{n}=x h(w[i . . j]) y, \\
& x \preceq_{s} h\left(a_{i-1}\right), \\
& y \preceq_{p} h\left(a_{j+1}\right), \\
& |x|,|y|<q,
\end{aligned}
$$

for some integers $i, j \geq 0$. If $x=y=\epsilon$, then using Lemma 8 , since $|z| \geq q$, the word $w[i . . j]$ contains an $n^{\prime}$ th power. Therefore $w$ contains an $n^{\prime}$ th power of length smaller than $\left|z^{n}\right|$, a contradiction. Now suppose that $x y \neq \epsilon$. Since $|z| \geq \frac{2 n q}{n}=2 q$, and $|x h(w[i])|,|h(w[j]) y|<2 q$, we can write

$$
\begin{aligned}
& x h(w[i]) \preceq_{p} z, \\
& h(w[j]) y \preceq_{s} z .
\end{aligned}
$$

Therefore $h(w[j]) y x h(w[i]) \preceq z^{2} \preceq z^{n}$. Since $h$ is synchronizing

$$
h(w[j]) y x h(w[i]) \preceq h(w[i . . j]) .
$$

Hence $y x=h(a)$ for some $a \in \Sigma$. Since $h$ is an SSM, we have either $a=a_{i-1}$ or $a=a_{j+1}$. Without loss of generality, suppose that $a=a_{i-1}$. Then we can write $h(w[i-1 . . j])=y x h(w[i . . j])$. The word $y x h(w[i . . j])$ is an $n$ 'th power, since it is a conjugate of $x h(w[i . . j]) y$. So we can write

$$
h(w[i-1 . . j])=z_{1}^{n}
$$

where $z_{1}$ is a conjugate of $z$. Note that $\left|z_{1}\right|=|z| \geq 2 q$. Now using Lemma 8 the word $w[i-1 . . j]$ contains an $n$ 'th power, and hence $w$ contains an $n^{\prime}$ th power of length smaller than $\left|z^{n}\right|$, a contradiction.

The following lemma states that, for an $\operatorname{SSM} h$ and a well-chosen word $w$, all circular $\left(\frac{13}{4}\right)^{+}$-powers in $h(w)$ are small.

Lemma 10. Let $h: \Sigma^{*} \rightarrow \Gamma^{*}$ be a strongly synchronizing q-uniform morphism. Let $w=a_{0} a_{1} a_{2} \cdots \in \Sigma^{\omega}$ be a circularly cubefree word. In addition, suppose that $w$ is squarefree. If $x_{1} t x_{2} \preceq h(w)$ for some words $t, x_{1}, x_{2}$, and $x_{2} x_{1}$ is a $(13 / 4)^{+}$-power, then $\left|x_{2} x_{1}\right|<22 q$.

Proof. The proof is by contradiction. Suppose there are words $t, x_{1}, x_{2}$, and $z$ in $\Gamma^{*}$ and a rational number $\alpha>\frac{13}{4}$ such that

$$
\begin{gathered}
x_{1} t x_{2} \preceq h(w) \\
\left|x_{2} x_{1}\right| \geq 22 q \\
x_{2} x_{1}=z^{\alpha} .
\end{gathered}
$$


Suppose $|z|<q$. Let $k$ be the smallest integer for which $\left|z^{k}\right| \geq q$. Then $\left|z^{k}\right|<2 q$, because otherwise $\left|z^{k-1}\right| \geq q$, a contradiction. We can write $x_{2} x_{1}=$ $\left(z^{k}\right)^{\beta}$, where $\beta=\frac{\left|x_{2} x_{1}\right|}{\left|z^{k}\right|}>\frac{22 q}{2 q}>\frac{13}{4}$. Therefore we can assume that $|z| \geq q$, since otherwise we can always replace $z$ with $z^{k}$, and $\alpha$ with $\beta$.

There are three cases to consider.

(a) Suppose that $x_{1}$ and $x_{2}$ are both long enough, so that each contains an image of a word under $h$. More formally, suppose that

$$
\begin{aligned}
& x_{1}=y_{1} h\left(w\left[i_{1} . . j_{1}\right]\right) y_{2}, \\
& x_{2}=y_{3} h\left(w\left[i_{2} . . j_{2}\right]\right) y_{4}, \\
& i_{1} \leq j_{1}, i_{2} \leq j_{2}, \\
& y_{1} \preceq_{s} h\left(a_{i_{1}-1}\right), \\
& y_{2} \preceq_{p} h\left(a_{j_{1}+1}\right), \\
& y_{3} \preceq_{s} h\left(a_{i_{2}-1}\right), \\
& y_{4} \preceq_{p} h\left(a_{j_{2}+1}\right), \\
& \left|y_{1}\right|,\left|y_{2}\right|,\left|y_{3}\right|, \text { and }\left|y_{4}\right|<q, \text { and } \\
& y_{2} t y_{3}=h\left(w\left[j_{1}+1 . . i_{2}-1\right]\right) .
\end{aligned}
$$

Let $v_{1}=w\left[i_{1} . . j_{1}\right]$ and $v_{2}=w\left[i_{2} . . j_{2}\right]$. See Fig 1$]$

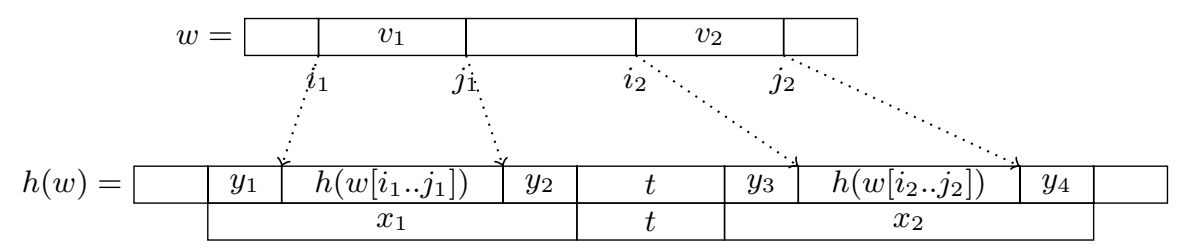

Fig. 1. $x_{1} t x_{2}$ is a factor of $h(w)$

There are two cases to consider.

(1) Suppose that $y_{4} y_{1}=\epsilon$. Let $v=w\left[i_{2} . . j_{2}\right] w\left[i_{1} . . j_{1}\right]$.

The word $h(v) y_{2}$ is a factor of $y_{3} h(v) y_{2}=z^{\alpha}$ of length $\geq 22 q-q=21 q$, and so

$$
h(v) y_{2}=z_{1}^{\beta},
$$

where $z_{1}$ is a conjugate of $z$, and $\beta \geq \frac{21}{22} \alpha>3$. Therefore we can write

$$
z_{1}^{3} \preceq_{p} h(v) y_{2} \preceq_{p} h\left(v w\left[j_{1}+1\right]\right) .
$$

Note that $\left|z_{1}\right|=|z| \geq q$, so using Lemma 8 , we can write $\left|z_{1}\right| \equiv 0(\bmod$ $q)$. Therefore

$$
z_{1}^{3} \preceq_{p} h(v) .
$$

Using Lemma 8 again, the word $v$ contains a cube, which means that the word $w$ contains a circular cube, a contradiction. 
(2) Suppose that $y_{4} y_{1} \neq \epsilon$. We show how to get two new factors $x_{1}^{\prime}=h\left(v_{1}^{\prime}\right) y_{2}^{\prime}$ and $x_{2}^{\prime}=y_{3}^{\prime} h\left(v_{2}^{\prime}\right)$, with $v_{1}^{\prime}, v_{2}^{\prime}$ nonempty, such that $x_{2}^{\prime} x_{1}^{\prime}=x_{2} x_{1}$. Then we use case (1) above to get a contradiction.

Let $s=h\left(w\left[j_{2}\right]\right) y_{4} y_{1} h\left(w\left[i_{1}\right]\right)$, and let $m$ be the smallest integer for which $\left|z^{m}\right| \geq|s|$. Note that if $|z|<|s|$, then

$$
\left|z^{m}\right|<2|s|<8 q .
$$

We show that at least one of the following inequalities holds:

$$
\begin{aligned}
& \left|h\left(v_{1}\right)\right| \geq q+\left|z^{m}\right|, \\
& \left|h\left(v_{2}\right)\right| \geq q+\left|z^{m}\right| .
\end{aligned}
$$

Suppose that both inequalities fail. Then using (11) and (2) we can write

$$
\left|x_{2} x_{1}\right|<2 q+2\left|z^{m}\right|+\left|y_{1} y_{2} y_{3} y_{4}\right|<6 q+2\left|z^{m}\right| .
$$

If $|z|<|s|$, then by (3) and (4) one gets $\left|x_{2} x_{1}\right|<22 q$, contradicting our assumption. Otherwise $|z| \geq|s|$, and hence $m=1$. Then

$$
\left|x_{2} x_{1}\right|=\alpha|z|<2 q+2|z|+\left|y_{1} y_{2} y_{3} y_{4}\right|<6 q+2|z|,
$$

and hence $|z|<6 q$. So $\left|x_{2} x_{1}\right|<6 q+2|z|<18 q$, contradicting our assumption. Without loss of generality, suppose that $\left|h\left(v_{1}\right)\right| \geq q+\left|z^{m}\right|$. Using the fact that $z$ is a period of $x_{2} x_{1}$, we can write

$$
h\left(v_{1}\right)\left[q+\left|z^{m}\right|-|s| . . q+\left|z^{m}\right|-1\right]=s,
$$

or, in other words,

$$
s \preceq h\left(v_{1}\right) .
$$

See Fig 2

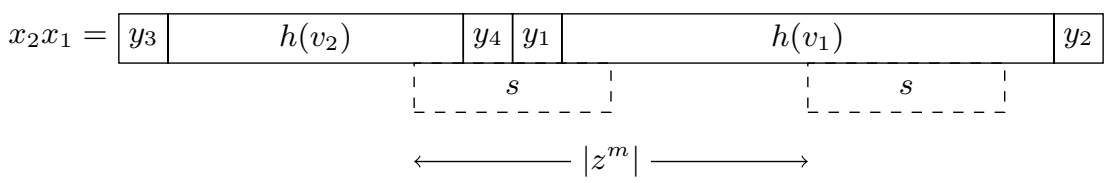

Fig. 2. $h\left(v_{1}\right)$ contains a copy of $s$

Using the fact that $h$ is synchronizing, we get that $y_{4} y_{1}=h(a)$ for some $a \in \Sigma$. Since $h$ is an SSM, we have either $a=a_{i_{1}-1}$ or $a=a_{j_{2}+1}$. Without loss of generality, suppose that $a=a_{j_{2}+1}$. Now look at the following factors of $h(w)$, which can be obtained from $x_{1}$ and $x_{2}$ by extending $x_{2}$ to the right and shrinking $x_{1}$ from the left:

$$
\begin{aligned}
& x_{1}^{\prime}=h\left(w\left[i_{1} . . j_{1}\right]\right) y_{2} \\
& x_{2}^{\prime}=y_{3} h\left(w\left[i_{2} . . j_{2}+1\right]\right) .
\end{aligned}
$$


See Fig 3

$h(w)=$\begin{tabular}{|c|c|c|}
\hline$\left|h\left(a_{i_{1}-1}\right)\right| h\left(a_{i_{1}} a_{i_{1}+1} \cdots a_{j_{1}}\right) \mid y_{2}$ & $t$ & $y_{3}\left|h\left(a_{i_{2}} a_{i_{2}+1} \cdots a_{j_{2}}\right)\right| h\left(a_{j_{2}+1}\right) \mid$ \\
\hline & $x_{1}^{\prime} \ldots \ldots \ldots$ & $x_{2}^{\prime} \ldots \ldots \ldots$
\end{tabular}

We can see that

Fig. 3. $x_{1}^{\prime}$ and $x_{2}^{\prime}$ are obtained from $x_{1}$ and $x_{2}$

$$
x_{2}^{\prime} x_{1}^{\prime}=y_{3} h\left(w\left[i_{2} . . j_{2}+1\right]\right) h\left(w\left[i_{1} . . j_{1}\right]\right) y_{2}=y_{3} h\left(w\left[i_{2} . . j_{2}\right]\right) y_{4} y_{1} h\left(w\left[i_{1} . . j_{1}\right]\right) y_{2}=x_{2} x_{1} .
$$

Now using case (1) we get a contradiction.

(b) Suppose that $x_{2}$ is too short to contain an image of a word under $h$. More formally, we can write

$$
x_{1}=y_{1} h(v) y_{2} \text { where }\left|x_{2}\right|<2 q \text { and }\left|y_{1}\right|,\left|y_{2}\right|<q
$$

for some words $y_{1}, y_{2} \in \Gamma^{*}$ and a word $v \preceq w$. Then $h(v)$ is a factor of $x_{2} x_{1}=z^{\alpha}$ of length $\geq 22 q-4 q=18 q$, and so

$$
h(v)=z_{1}^{\beta}
$$

where $z_{1}$ is a conjugate of $z$, and $\beta \geq \frac{18}{22} \alpha>2$. Note that $\left|z_{1}\right|=|z| \geq q$, so using Lemma 8 , the word $v$ contains a square, a contradiction.

(c) Suppose that $x_{1}$ is not long enough to contain an image of a word under $h$. An argument similar to (b) applies here to get a contradiction.

The following 15-uniform morphism is an example of an SSM:

$$
\begin{aligned}
& \mu(0)=012102120102012 \\
& \mu(1)=201020121012021 \\
& \mu(2)=012102010212010 \\
& \mu(3)=201210212021012 \\
& \mu(4)=102120121012021 \\
& \mu(5)=102010212021012 .
\end{aligned}
$$


Another example of an SSM is the 4-uniform morphism $\psi: \Sigma_{6}^{*} \rightarrow \Sigma_{6}^{*}$ as follows:

$$
\begin{aligned}
& \psi(0)=0435 \\
& \psi(1)=2341 \\
& \psi(2)=3542 \\
& \psi(3)=3540 \\
& \psi(4)=4134 \\
& \psi(5)=4105 .
\end{aligned}
$$

Our goal is to show that $\mu\left(\psi^{\omega}(0)\right)$ is circularly $\left(\frac{13}{4}\right)^{+}$-power-free. For this purpose, we first prove that $\psi^{\omega}(0)$ is circularly cubefree. Then we apply Lemma 10. for $h=\mu$ and $w=\psi^{\omega}(0)$.

Lemma 11. The fixed point $\psi^{\omega}(0)$ is squarefree.

Proof. Suppose that $\psi^{\omega}(0)$ contains a square. Using Lemma 9, there is a square $z z \preceq \psi^{\omega}(0)$ such that $|z z|<16$. Using a computer program, we checked all factors of length smaller than 16 in $\psi^{\omega}(0)$, and none of them is a square. This is a contradiction.

Lemma 12. The fixed point $\psi^{\omega}(0)$ is circularly cubefree.

Proof. By contradiction. Let $w=a_{0} a_{1} a_{2} \cdots=\psi^{\omega}(0)$. Suppose $x_{1} t x_{2} \preceq w$, and $x_{2} x_{1}=z^{3}$ for some words $t, x_{1}, x_{2}, z$, where

$$
\begin{aligned}
& x_{1}=y_{1} \psi\left(w\left[i_{1} . . j_{1}\right]\right) y_{2}, \\
& x_{2}=y_{3} \psi\left(w\left[i_{2} . . j_{2}\right]\right) y_{4}, \\
& y_{1} \preceq_{s} \psi\left(a_{i_{1}-1}\right), \\
& y_{2} \preceq_{p} \psi\left(a_{j_{1}+1}\right), \\
& y_{3} \preceq_{s} \psi\left(a_{i_{2}-1}\right), \\
& y_{4} \preceq_{p} \psi\left(a_{j_{2}+1}\right), \\
& \left|y_{1}\right|,\left|y_{2}\right|,\left|y_{3}\right|, \text { and }\left|y_{4}\right|<4, \\
& y_{2} t y_{3}=\psi\left(w\left[j_{1}+1 . . i_{2}-1\right]\right),
\end{aligned}
$$

for proper choices of the integers $i_{1}, i_{2}, j_{1}, j_{2}$. Let $v_{1}=w\left[i_{1} . . j_{1}\right]$ and $v_{2}=w\left[i_{2} . . j_{2}\right]$.

Using a computer program, we searched for circular cubes of length not greater than 66, and it turns out that there is no such circular cube in $w$. Thus we can assume that $\left|x_{2} x_{1}\right|>66$ so $|z|>22$. Moreover suppose that $x_{2} x_{1}$ has the smallest possible length.

There are two cases to consider.

(a) Suppose that $y_{4} y_{1}=\epsilon$. If $y_{2} y_{3}=\epsilon$, then $\psi\left(v_{2} v_{1}\right)=z^{3}$. Using Lemma 8, we get that $v_{2} v_{1}$ contains a cube. Hence $w$ contains a smaller circular cube, a contradiction. 
Suppose that $y_{2} y_{3} \neq \epsilon$. Since $\left|y_{3} \psi\left(w\left[i_{2}\right]\right)\right|,\left|\psi\left(w\left[j_{1}\right]\right) y_{2}\right|<8$ and $|z|>22$, we can write

$$
\begin{aligned}
& y_{3} \psi\left(w\left[i_{2}\right]\right) \preceq_{p} z, \\
& \psi\left(w\left[j_{1}\right]\right) y_{2} \preceq_{s} z .
\end{aligned}
$$

Therefore $\psi\left(w\left[j_{1}\right]\right) y_{2} y_{3} \psi\left(w\left[i_{2}\right]\right) \preceq z^{3}$, and since $\psi$ is synchronizing

$$
\psi\left(w\left[j_{1}\right]\right) y_{2} y_{3} \psi\left(w\left[i_{2}\right]\right) \preceq \psi\left(v_{2} v_{1}\right) .
$$

Hence $y_{2} y_{3}=\psi(b)$ for some $b \in \Sigma_{6}$. Since $\psi$ is an SSM, we have either $b=a_{i_{2}-1}$, or $b=a_{j_{1}+1}$. Without loss of generality, suppose that $b=a_{i_{2}-1}$. So we can write

$$
\psi\left(w\left[i_{2}-1 . . j_{2}\right] w\left[i_{1} . . j_{1}\right]\right)=y_{2} y_{3} \psi\left(w\left[i_{2} . . j_{2}\right] w\left[i_{1} . . j_{1}\right]\right) .
$$

The word $y_{2} y_{3} \psi\left(v_{2} v_{1}\right)$ is a cube, since it is a conjugate of $y_{3} \psi\left(v_{2} v_{1}\right) y_{2}$. So we can write

$$
\psi\left(w\left[i_{2}-1 . . j_{2}\right] w\left[i_{1} . . j_{1}\right]\right)=z_{1}^{3}
$$

where $z_{1}$ is a conjugate of $z$. Then using Lemma 8 , the word $w\left[i_{2}-1 . . j_{2}\right] w\left[i_{1} . . j_{1}\right]$ contains a cube. Note that since $y_{2} y_{3} \neq \epsilon$ we have $j_{1}<i_{2}-1$. Hence $w\left[i_{2}-1 . . j_{2}\right] w\left[i_{1} . . j_{1}\right]$ is a circular cube of $w$, a contradiction.

(b) Suppose that $y_{4} y_{1} \neq \epsilon$. We show how to get two new factors $x_{1}^{\prime}=h\left(v_{1}^{\prime}\right) y_{2}^{\prime}$ and $x_{2}^{\prime}=y_{3}^{\prime} h\left(v_{2}^{\prime}\right)$ of $w$, for nonempty words $v_{1}^{\prime}, v_{2}^{\prime}$, such that $x_{2}^{\prime} x_{1}^{\prime}=x_{2} x_{1}$. Then we use case (a) above to get a contradiction.

The word $w$ is squarefree due to Lemma [1], Therefore $\left|x_{1}\right|,\left|x_{2}\right|>|z|>\frac{66}{3}$ and hence $\left|v_{1}\right|,\left|v_{2}\right|>0$. One can observe that either $\left|\psi\left(v_{1}\right)\right| \geq 4+|z|$ or $\left|\psi\left(v_{2}\right)\right| \geq 4+|z|$. Without loss of generality, suppose that $\left|\psi\left(v_{1}\right)\right| \geq 4+|z|$. Let $s=w\left[j_{2}\right] y_{4} y_{1} w\left[i_{1}\right]$. Now, using the fact that $z$ is a period of $x_{2} x_{1}$, we can write

$$
\psi\left(v_{1}\right)[4+|z|-|s| . .4+|z|-1]=s,
$$

or, in other words,

$$
s \preceq \psi\left(v_{1}\right) .
$$

Using the fact that $\psi$ is synchronizing, we get that $y_{4} y_{1}=\psi(a)$ for some $a \in \Sigma_{6}$. Since $\psi$ is an SSM, we have either $a=a_{i_{1}-1}$, or $a=a_{j_{2}+1}$. Without loss of generality, suppose that $a=a_{j_{2}+1}$. Now look at the following factors of $w$, which can be obtained from $x_{1}$ and $x_{2}$ by extending $x_{2}$ to the right and shrinking $x_{1}$ from the left

$$
\begin{aligned}
& x_{1}^{\prime}=\psi\left(w\left[i_{1} . . j_{1}\right]\right) y_{2} \\
& x_{2}^{\prime}=y_{3} \psi\left(w\left[i_{2} . . j_{2}+1\right]\right) .
\end{aligned}
$$

We can write

$$
x_{2}^{\prime} x_{1}^{\prime}=y_{3} \psi\left(w\left[i_{2} . . j_{2}+1\right]\right) \psi\left(w\left[i_{1} . . j_{1}\right]\right) y_{2}=y_{3} \psi\left(v_{2}\right) y_{4} y_{1} \psi\left(v_{1}\right) y_{2}=x_{2} x_{1}=z^{3} .
$$

So using case (a) we get a contradiction. 
Theorem 13. $\operatorname{RTC}(3)=\frac{13}{4}$.

Proof. First let us show that RTC(3) $\geq \frac{13}{4}$.

Suppose there exists an infinite word $w$ that avoids circular $\alpha$-powers, for $\alpha<4$. We now argue that for every integer $C$, there exists an infinite word $w^{\prime}$ that avoids both squares of length $\leq C$ and circular $\alpha$-powers. Note that none of the factors of $w$ looks like $x x y x x$, since $w$ avoids circular 4-powers. Therefore, every square in $w$ occurs only finitely many times. Therefore $w^{\prime}$ can be obtained by removing a sufficiently long prefix of $w$.

Computer search verifies that the longest circularly $\frac{13}{4}$-power-free word over a 3-letter alphabet that avoids squares $x x$ where $|x x|<147$ has length 147 . Therefore the above argument for $C=147$ shows that circular $\frac{13}{4}$-powers are unavoidable over a 3-letter alphabet.

Now to prove $\operatorname{RTC}(3)=\frac{13}{4}$, it is sufficient to give an example of an infinite word that avoids circular $\left(\frac{13}{4}\right)^{+}$-powers. We claim that $\mu\left(\psi^{\omega}(0)\right)$ is such an example. We know that $\psi^{\omega}(0)$ is circularly cubefree. Therefore we can use Lemma 10 for $w=\psi^{\omega}(0)$ and $h=\mu$. So if $x t y \preceq \mu\left(\psi^{\omega}(0)\right)$, and $y x$ is a $\left(\frac{13}{4}\right)^{+}$-power, then $|y x|<22 \times 15$. Now there are finitely many possibilities for $x$ and $y$. Using a computer program, we checked that none of them leads to a $\left(\frac{13}{4}\right)^{+}$-power. This completes the proof.

\section{Another Interpretation}

We could, instead, consider the supremum of $\exp (p)$ over all products of $i$ factors of $w$. Call this quantity $\operatorname{pexp}_{i}(w)$.

Proposition 14. If $w$ is a recurrent infinite word, then $\operatorname{pexp}_{2}(w)=\operatorname{cexp}(w)$.

Proof. Let $s$ be a product of two factors of $w$, say $s=x y$. Let $y$ occur for the first time at position $i$ of $w$. Since $w$ is recurrent, $x$ occurs somewhere after position $i+|y|$ in $w$. So there exists $z$ such that $y z x$ is a factor of $w$. Then $x y$ is a factor of a conjugate of a factor of $w$.

On the other hand, from Proposition 2, we know that if $s$ is a conjugate of a factor of $w$, then $s=v t$ where $t u v$ is a factor of $w$. Then $s$ is the product of two factors of $w$.

We can now study the repetition threshold for $i$-term products, $\mathrm{RT}_{i}(k)$, which is the infimum of $\operatorname{pexp}_{i}(w)$ over all words $w \in \Sigma_{k}^{\omega}$. Note that

$$
\mathrm{RT}_{2}(k) \geq \operatorname{RTC}(k) .
$$

The two recurrent words, the Thue-Morse word and $\mu\left(\psi^{\omega}(0)\right)$, introduced in Section 4, are circularly RTC $(2)^{+}$-power-free and circularly RTC $(3)^{+}$-power-free, respectively. Using Proposition 14, we get that $\mathrm{RT}_{2}(k)=\mathrm{RTC}(k)$ for $k=2,3$.

Theorem 15. For $i \geq 1$ we have $\mathrm{RT}_{i}(2)=2 i$. 
Proof. From Thue we know there exists an infinite $2^{+}$-power-free word. If some product of factors $x_{1} x_{2} \cdots x_{i}$ contains a $(2 i)^{+}$-power, then some factor contains a $2^{+}$-power, a contradiction. $\operatorname{So~}_{\mathrm{RT}_{i}}(2) \leq 2 i$.

For the lower bound, fix $i \geq 2$, and let $w \in \Sigma_{2}^{\omega}$ be an arbitrary word. Either 00 or 11 appears infinitely often, or $w$ ends in a suffix of the form $(01)^{\omega}$. In the latter case we get arbitrarily high powers, and the former case there is a product of $i$ factors with exponent $2 i$.

It would be interesting to obtain more values of $\mathrm{RT}_{i}(k)$. We propose the following conjectures which are supported by numerical evidence:

$$
\begin{aligned}
& \operatorname{RT}_{2}(4)=\operatorname{RTC}(4)=\frac{5}{2}, \\
& \operatorname{RT}_{2}(5)=\operatorname{RTC}(5)=\frac{105}{46}, \text { and } \\
& \operatorname{RT}_{2}(k)=\operatorname{RTC}(k)=1+\operatorname{RT}(k)=\frac{2 k-1}{k-1} \text { for } k \geq 6 .
\end{aligned}
$$

We know that the values given above are lower bounds for $\mathrm{RTC}(k)$.

\section{References}

1. A. Aberkane and J. D. Currie. There exist binary circular $5 / 2^{+}$power free words of every length. Electronic J. Combin. 11 (1), 2004, Paper \#R10. Available at http://www1.combinatorics.org/Volume_11/Abstracts/v11i1r10.html

2. F.-J. Brandenburg. Uniformly growing k-th power-free homomorphisms. Theoret. Comput. Sci. 23 (1983), 69-82.

3. A. Carpi. On Dejean's conjecture over large alphabets. Theoret. Comput. Sci. $\mathbf{3 8 5}$ (2007), 137-151.

4. J. Currie and N. Rampersad. A proof of Dejean's conjecture. Math. Comp. 80 (2011), 1063-1070.

5. F. Dejean. Sur un théorème de Thue. J. Combin. Theory. Ser. A 13 (1972), 90-99.

6. I. A. Gorbunova. Repetition threshold for circular words. Electronic J. Combin. $\mathbf{1 9}$ (4), Paper \#11. Available at http://www.combinatorics.org/ojs/index.php/eljc/article/view/v19i4p11.
T. Harju and D. Nowotka. Cyclically repetition-free words on small alphabets. Inform Process Lett. 110 (2010) 591-595.

8. L. Ilie, P. Ochem, and J. Shallit. A generalization of repetition threshold. Theoret. Comput. Sci. 345 (2005), 359-369.

9. M. Rao. Last cases of Dejean's conjecture. Theoret. Comput. Sci. 412 (2011), 3010-3018.

10. A. Thue. Über unendliche Zeichenreihen. Norske vid. Selsk. Skr. Mat. Nat. Kl. 7 (1906), 1-22. Reprinted in Selected Mathematical Papers of Axel Thue, T. Nagell, ed., Universitetsforlaget, Oslo, 1977, pp. 139-158.

11. A. Thue. Über die gegenseitige Lage gleicher Teile gewisser Zeichen reihen. Norske vid. Selsk. Skr. Mat. Nat. Kl. 1 (1912), 1-67. Reprinted in Selected Mathematical Papers of Axel Thue, T. Nagell, ed., Universitetsforlaget, Oslo, 1977, pp. 413-478. 